COMO CITAR ESTE ARTÍCULO:

Fuentealba-Martínez, M.S., González-Ramírez, I.X. y Valdebenito-Larenas, C. (2018). Un novedoso instrumento para evaluar la calidad de la mediación de conflictos jurídicos familiares en Chile. Revista Jurídicas, 15 (1), 65-87.

DOI: 10.17151/jurid.2018.15.1.5.

Recibido el 15 de junio de 2016 Aprobado el 4 de octubre de 2017

\section{UN NOVEDOSO INSTRUMENTO PARA EVALUAR LA CALIDAD DE LA MEDIACIÓN DE CONFLICTOS FAMILIARES EN CHILE}

\author{
María Soledad Fuentealba-Martínez* \\ IsABel Ximena González-Ramírez** \\ Caterine Valdebenito-Larenas***
}

\section{RESUMEN}

El presente artículo trata del diseño, aplicación y análisis de un novedoso instrumento para la evaluación de procesos de mediación de conflictos jurídicos en materia de familia, contemplado como uno de los productos del avance en el proceso de mejoramiento continuo de la calidad de la mediación familiar licitada, lo que es de relevancia al no existir estudios e instrumentos validados, nacionales o internacionales para medir la calidad de la mediación. Utilizamos una metodología empírica, con un diseño exploratorio, evaluando los resultados de dos estudios realizados durante cuatro años al universo total de mediadores familiares, concluyendo que el trabajo de supervisión y retroalimentación mejora efectivamente la calidad de la mediación.
Palabras clave: mediación familiar, calidad, supervisión, instrumentos de evaluación.

Magíster en gestión colaborativa de conflictos: Mediación. Facultad de Derecho de la Universidad Central de Chile, Santiago de Chile. Cursa Doctorado en Derecho en la Universidad de Buenos Aires, Argentina. E-mail:mfuentealbam@ucentral.cl.Google Scholar. ORCID: 0000-0001-8232-082. Researchgate: https://www.researchgate. net/profile/Maria_Fuentealba2. Academia: https:/ucentral. academia.edu/MariaSoledadFuentealbaMartinez

** Doctora en Derecho, de la Universidad de Buenos Aires Argentina. E-mail: igonzalezr@ucentral.cl. Google Scholar. ORCID: 0000-0001-6845-8198. Researchgate: https://www.researchgate.net/profile/lsabel_Gonzalez_ Ramirez. Academia: https://ucentral.academia.edu/ ISABELXIMENAGONZALEZRAMIREZ.

**** Magíster en Gestión colaborativa de conflictos: Mediación. Cursa Doctorado en Derecho en Universidad de Buenos Aires, Argentina. E-mail: cvaldevenitol@ucentral.cl. Google Scholar. ORCID: 0000-0002-3684-0066. 


\section{AN ORIGINAL TOOL TO ASSESS \\ THE QUALITY OF FAMILY LAW \\ DISPUTES MEDIATION IN CHILE}

\section{ABSTRACT}

This article deals with the design, implementation and analysis of a new instrument for the assessment of processes of mediation of legal disputes in family matters, considered as one of the products of progress in the process of continuous improvement of the quality of family mediation which is relevant in the absence of studies and validated, national or international instruments to measure the quality of mediation. An empirical methodology with an exploratory design was used, evaluating the results of two studies conducted over four years which dealt with the total universe of family mediators and concluding that the work of monitoring and feedback, effectively improve quality of mediation.

KEY wORDs: family mediation, quality, supervision, evaluation instruments. 


\section{INTRODUCCIÓN}

El presente artículo referido a uno de los principales productos del avance que ha tenido Chile en cuanto a la calidad de mediación familiar licitada desde su incorporación obligatoria y previa, tiene por objeto mostrar los resultados de los estudios realizados por la Unidad de Mediación del Ministerio de Justicia y Derechos Humanos ${ }^{1}$ a través del soporte técnico de la Universidad Central de Chile ${ }^{2}$, sobre el sistema de supervisión de la calidad en los procesos de mediación familiar efectuados los años 2012-2013 y 2014-2015.

Dichos estudios permitieron validar un sistema de supervisión y los instrumentos necesarios para medir y comparar los distintos componentes propios de la calidad en los procesos de mediación familiar de cada profesional licitado, centro de mediación y región del país, retroalimentando su gestión con el propósito de mejorar la calidad del servicio.

Las investigaciones antes descritas, se enmarcan en el siguiente contexto: Con la reforma a la Ley de Tribunales de Familia, y la entrada en vigencia de la Ley $\mathrm{N}^{\mathrm{o}}$ 20.286 de 2008 que introduce mejoras orgánicas y procedimentales a la Ley $\mathrm{N}^{\mathrm{o}}$ 19.968 de 2004 que crea los Tribunales de Familia, se incorpora la mediación familiar $^{3}$ previa, respecto de determinadas materias, establecida en el art. 106 de la referida ley, relativas al derecho de alimentos, cuidado personal y al derecho de los padres e hijos e hijas que vivan separados a mantener una relación directa y regular, aún en el marco de una acción de divorcio o separación judicial (Acuña, 2011); materias que son más usuales en tribunales de familia.

Para estos efectos, se establece la obligatoriedad de que las partes intenten previamente a la interposición de una acción judicial, un acuerdo a través de un proceso de mediación (Stein, 2015).

Es así como la mediación, al ser uno de los mecanismos que el Estado ofrece a las personas para la solución jurídica de importantes conflictos (Preyer, 2015, p. 2), requiere que sus servicios sean de calidad, surgiendo por ello, el siguiente planteamiento del problema: ¿cómo es el sistema que tiene Chile para supervisar

\footnotetext{
${ }^{1}$ En adelante UMED.

2 En adelante UCEN.

${ }^{3}$ De acuerdo con el art. 103 de la ley que crea los tribunales de familia, se entiende por mediación aquel sistema de resolución de conflictos en el que un tercero imparcial, sin poder decisorio, llamado mediador, ayuda a las partes a buscar por sí mismas una solución al conflicto y sus efectos, mediante acuerdos. Asimismo, la mediación familiar ha sido definida como "Un proceso de resolución de conflictos regulado por principios dogmáticos y legales en el cual, un (a) mediador (a) imparcial, genera comunicación efectiva y constructiva entre las partes y promueve la participación igualitaria, autónoma y responsable en la búsqueda de soluciones a conflictos familiares, facilitando la generación de acuerdos construidos desde las partes interesadas con recíproca satisfacción subjetiva". Informe Final definitivo, Auditoría externa para la evaluación de la Calidad de los Procesos de Mediación Familiar, Centro de Estudios y Asistencia Legislativa de la Pontificia Universidad Católica de Valparaíso, 2011.
} 
la calidad de los servicios de mediación familiar licitados que ofrece el Estado y cuáles son los estándares de calidad obtenidos por sus mediadores en la aplicación de dicho sistema de supervisión?

Este estudio se plantea como hipótesis principal que: Chile es uno de los países de Latinoamérica que cuenta con un sistema probado de supervisión de la calidad del servicio de mediación familiar licitado, aplicado periódicamente a sus mediadores, cuyos procesos e instrumentos están en permanente actualización. Y como hipótesis subsidiaria: los estándares de calidad de los mediadores licitados en Chile están dentro de un parámetro de calidad alta.

Ahora bien, podemos afirmar que el estado de la cuestión en esta materia demuestra que prácticamente no existen sistemas estandarizados de evaluación de la calidad de mediadores familiares, en otros países. No obstante, en países como Argentina se hace alusión en la ley a competencias que deben presentar los mediadores familiares, tales como confiable, buen oyente, perceptivo, debe poseer capacitación específica y entrenamiento en el tratamiento interdisciplinario de una problemática, poseedor de una seria intención de ayudar, hábil para la comunicación, flexible, neutral, imparcial, sigiloso (respecto de la confidencialidad y de las normas éticas que le impiden violar cualquier secreto) creativo, paciente y conciliador (García y Aguilera, 2003) y en España, "se realizan procesos de supervisión, que contemplan trabajo clínico y análisis de casos" (Migrainfo, 2008, p. 28), pero en materia de mediación comunitaria.

Por otro lado, el cuestionamiento acerca de la calidad de los procesos de mediación se ha instado en el marco de reflexiones teóricas llevadas a la práctica, como es el caso de Brandoni (2011), quien plantea interrogantes como: ¿Qué mecanismos pueden instrumentarse para construir, cuidar y mejorar la calidad de la tarea del mediador? ¿Cómo se establecen los estándares de calidad? ¿Quién y cómo se mide la calidad del servicio de mediación y la tarea de los mediadores? Se presentan propuestas en líneas generales, apropiadas para que cada uno de los operadores de justicia, diseñen e implementen mecanismos acordes a su ejercicio profesional, lo que da cuenta de una carencia de sistematicidad en el abordaje de la calidad de aquellos procesos de solución colaborativa de conflictos jurídicos familiares en gran parte de los países.

El caso de España se presenta como antagónico al de Chile, ya que en diversas comunidades autónomas españolas se ha regulado la mediación de conflictos desde la promulgación de la recomendación del Consejo de Europa sobre la utilización de vías alternativas de resolución de conflictos el 16 de septiembre de 1986, considerando que comunidades como Cataluña, Valencia, Galicia, entre otras, emitieron instrumentos legislativos tendientes a regular en mayor o menor medida, la solución colaborativa de conflictos como vía alternativa para prevenir y 
reducir la excesiva carga de los tribunales de justicia (López, 2016); lo que deriva en el planteamiento sobre la relevancia de la calidad de dichos procesos, como mecanismo para generar confianza en la mediación como vía alternativa a la administración de justicia, en la medida en que se mantenga el equilibrio entre los derechos garantizados legalmente y las necesidades de las partes.

\section{EVALUACIÓN DE LA MEDIACIÓN FAMILIAR EN CHILE}

En Chile, para garantizar la imparcialidad y calidad del ejercicio profesional de los mediadores, se creó un Sistema Nacional de Mediación licitado ${ }^{4}$, cuya gestión y administración corresponde a la Unidad de Mediación del Ministerio de Justicia ${ }^{5}$, conforme lo establecido en la Ley N 19.968 de 2004, destinadas a la contratación de los servicios de mediadores familiares, que garanticen la adecuada cobertura de estos servicios a nivel nacional (MINJU, 2010).

Asimismo, el Ministerio de Justicia ha establecido un conjunto de acciones técnicas, destinadas a la evaluación de la calidad del servicio prestado por los mediadores "con el fin de evaluar y perfeccionar los servicios de mediación, con miras a alcanzar estándares adecuados de calidad" (Correa, 2014, p. 119).

De esta manera, la elaboración y prueba del instrumento denominado "Evaluación de la calidad del proceso de mediación familiar" ${ }^{\prime \prime}$, puede considerarse una innovadora iniciativa de la UMED del MINJU, que constituye un importante avance en un proceso iniciado hace ya unos años por esta unidad, en pos del mejoramiento de la calidad de este servicio (Rawls, 2002), la que se suma a otros procesos, como las pruebas de conocimientos aplicadas a estos mediadores a nivel nacional, evaluándolos periódicamente, para medir su mejoría continua.

La importancia de un proceso de supervisión garantiza que el Estado ejerza control y dirección de modelos de desempeño, útiles para dar cumplimiento al mandato legal de brindar servicios de mediación familiar de buena calidad y rescatar criterios de supervisión clínica (Universidad de Concepción, 2006).

Esta supervisión clínica tiene por objeto observar y evaluar la práctica de la mediación familiar licitada, con base en la metodología $\mathrm{ECAME}^{7}$, que permite proponer mejoras en la práctica de los procesos de mediación, de las instituciones

\footnotetext{
${ }^{4}$ En adelante SNM.

${ }^{5}$ En adelante MINJU.

${ }^{6}$ En adelante ECAME.

${ }^{7}$ Se refiere al estudio de la Evaluación de la Calidad de los Procesos de Mediación Familiar, realizado por la CEAL. PUCV en el año 2011, que es conocido por la sigla del instrumento de evaluación ECAME, y que da base al desarrollo de esta investigación aplicada nacionalmente.
} 
privadas con que contrata el Estado ${ }^{8}$, todo lo cual se reflejó en los resultados en los procesos licitatorios: 2011 (PUCV), 2013 y 2015 (UCEN).

Para estos efectos, conforme a la escuela nórdica, se define 'calidad' en cuanto a dos dimensiones: calidad del proceso, entendida como aquella juzgada por el consumidor (usuario en el proceso de mediación) durante la prestación del servicio y engloba las calidades técnica (entre las que estarían la formación y la acreditación profesional) y funcional (respeto a las normas del proceso, a códigos deontológicos y éticos) (Lehtinen \& Lehtinen, 1982) y de resultados, definida como aquella que es juzgada por el consumidor después de la prestación del servicio y puede ser objeto de influencias externas al proceso, por lo que su control es más difícil de determinar con parámetros cuantitativos (por ejemplo, número de acuerdos alcanzados) (Lehtinen \& Lehtinen, 1982).

No obstante, un tercer elemento determinante para la calidad se encuentra en las competencias y capacidades del mediador, "obteniendo resultados que dan mayor satisfacción a los usuarios, operadores e instituciones" (Aguilar, 1994, p. 29), instalando una mirada sistémica a la práctica del mediador y promoviendo por el Estado chileno, el mejoramiento continuo en sus prácticas y condiciones laborales, que impacten a los ciudadanos.

Para ello, se implementó una metodología mixta de carácter cuantitativa y cualitativa, de observación participante, que consiste en la evaluación y medición, mediante la aplicación de la pauta ECAME, de conductas definidas previamente a desarrollar por los mediadores dentro de sala de mediación (Serrano, López, Rodríguez y Mirón, 2006), de manera similar para todos los participantes en este estudio, para terminar con una autoevaluación del/a mediador/a y la retroalimentación que devuelve a los mediadores.

\section{Resultados ECAME período 20I2-20 I3}

En este contexto, es que el Centro de Mediación, Negociación y Arbitraje de la Universidad Central de Chile $^{9}$, trabajó en dos proyectos de investigación aplicada propuestos por el MINJU, durante cuatro años, por medio de licitaciones públicas, con la finalidad de diseñar e implementar un sistema de supervisión clínica de la práctica de mediación familiar licitada, que pueda evaluar y apoyar el fortalecer su desempeño, mediante un instrumento único validado metodológicamente y por expertos, que pueda ser replicado.

\footnotetext{
${ }^{8}$ Para ello, revisar el link http://mediacionchile.cl, que contiene las distintas auditorías de evaluación de satisfacción del usuario en mediación, realizadas desde 2006 en adelante.

${ }^{9}$ En adelante CMNA.
} 


\section{Antecedentes metodológicos}

De esta forma, los objetivos de la primera investigación denominada "Auditoría de la Calidad de los Servicios de la Mediación Familiar Licitada", realizada por la UCEN en los años 2012-2013, consistieron en: 1.- Diseñar un sistema nacional de gestión de supervisiones clínicas para mediadores. 2.- Implementar la aplicación de la metodología ECAME de supervisión 3.- Sistematizar cuantitativa y cualitativamente los resultados obtenidos. 4.- Generar un proceso de retroalimentación y perfeccionamiento del desempeño profesional, estableciendo criterios de calidad compartidos (Muñoz, 2015); y 5.- Realizar propuestas de mejoramiento al instrumento y a la metodología ECAME.

Esta investigación de corte evaluativo propuso para su realización, utilizar metodología de carácter mixto. Con un enfoque cuantitativo de interpretación de los resultados numéricos y estadísticos del proceso de supervisión, que permitió resultados seleccionados por género, mediador, centro de mediación, zona de licitación y por región, junto a la evaluación estadística. Todo esto, incorporando un enfoque cualitativo en la recolección y tratamiento de los aspectos de observación y de autoevaluación, propios de la supervisión y reuniones clínicas a los mediadores.

El estudio tuvo cobertura en todo el territorio nacional, dividido en cuatro macrozonas: norte, sur, centro y metropolitana, realizándose la supervisión en las trece ciudades capitales regionales de Chile, a 279 mediadores, equivalente a un $90,58 \%$ del total de mediadores contratados por el MINJU, por un equipo interdisciplinario de mediadores expertos del sector privado y público (Beloff, 2010).

La Pauta ECAME: este instrumento fue diseñado y validado en 2011 por el equipo de la CEAL-PUCV, con el objeto de evaluar la calidad de los procesos de mediación familiar de los centros contratados por el Ministerio de Justicia (CEAL-PUCV. 2011), a través de la estandarización de cuatro componentes, expresos en la Tabla 1.

Tabla 1. Recursos metodológicos.

\begin{tabular}{ll}
\hline Componentes de proceso & Concepto \\
\hline Legitimidad & $\begin{array}{l}\text { Características que determinan la validez de los procesos de } \\
\text { mediación familiar según principios legales y dogmáticos. }\end{array}$ \\
\hline Proceso comunicacional & $\begin{array}{l}\text { Características que permiten el entendimiento y la } \\
\text { interacción positiva de entre las partes en el proceso de } \\
\text { mediación familiar. }\end{array}$
\end{tabular}




\begin{tabular}{ll} 
Proceso participativo & $\begin{array}{l}\text { Características que permiten la participación activa e } \\
\text { igualitaria de las partes en el proceso de mediación familiar. }\end{array}$ \\
\hline Proceso resolutivo & $\begin{array}{l}\text { Características que definen un procedimiento colaborativo } \\
\text { de resolución de conflictos familiares. }\end{array}$
\end{tabular}

Fuente: CEAL-PUCV. Auditoría Externa para la Calidad de los Procesos de Mediación Familiar. Informe Final 2011.

Para ello, se contó con:

a. Pauta de autoevaluación: creado en 2012 por el equipo de la UCEN, con el enfoque teórico "supervisión entre pares", que es el "espacio de trabajo entre un/a mediador/a y otro/a, destinado a la reflexión del trabajo realizado en la sala de mediación, por uno/a de ellos/as, convirtiendo la experiencia en un aprendizaje significativo" (Sallard, 2009, p. 39). Para ello, se usó la misma lógica analítica y temática de la pauta ECAME, con una pauta de evaluación numérica de 1,0 a 7,0.

b. Pauta de retroalimentación: instrumento creado por el equipo de la UCEN en 2012, de corte cualitativo agrupados en torno a los cuatro componentes del ECAME, en los que el supervisor comparte con el/a mediador/a sus impresiones técnicas de la práctica observada, relevando un FODA, para su aprendizaje y reflexión.

c. Plan de análisis de medidas de tendencia central con base en porcentajes: para el caso de las variables relativas a caracterización de mediadores(as) y del proceso de supervisión, junto a los ítems del ECAME. Posteriormente, se procedió al cálculo de las variables agregadas por componente, para lo que se identificaron los ítems correspondientes a cada uno de los componentes de la metodología ECAME, sumándose las puntuaciones de un componente, para luego dividirlo por la suma total de la puntuación máxima esperada para dicho componente y finalmente multiplicarlo por cien:

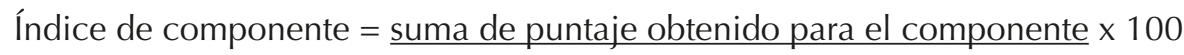
suma de puntaje máximo esperado para el componente

Una vez calculados los índices por componentes, se llevó a cabo el cálculo del índice general de calidad de la mediación familiar, sumando los índices por variable para luego dividir el resultado por cuatro.

Índice general de calidad de mediación familiar = $\underline{\text { suma de índices por componente }}$ 
Con la obtención de los índices por componente y del Índice General de Calidad de la Mediación Familiar, se posibilita el análisis bivariado -derivado del cruce de dos variables- de datos (Hernández y Sampieri, 2003), la prueba T para muestras independientes y la prueba U de Mann-Whitney (Kerlinger, 2002). Se realizó también el análisis porcentual con base en tablas de contingencia, de la asociación entre las variables agregadas tipo de término del proceso de mediación y su duración.

Junto a los análisis ya mencionados, se generó una variable ordinal de resultado del Índice General de Calidad de la Mediación Familiar, evidente en la Tabla 2.

Tabla 2. Categorías de calidad de la mediación.

\begin{tabular}{ll}
\hline Categoría & Índice de calidad de la mediación \\
\hline Calidad muy baja & $0 \%-20 \%$ \\
\hline Calidad baja & $21 \%-40 \%$ \\
\hline Calidad media & $41 \%-60 \%$ \\
\hline Calidad alta & $61 \%-80 \%$ \\
\hline Calidad muy alta & $81 \%-100 \%$ \\
\hline
\end{tabular}

Fuente: PUCV/CEAL, 2011. "Auditoría Externa para la Evaluación de la Calidad de los Procesos de Mediación Familiar".

\section{Ejecución del proyecto:}

Se cubrió el $100 \%$ del territorio el primer trimestre de 2013. Los/as mediadores dispusieron de 120 minutos para su participación en este proceso evaluativo complejo, compuesto de tres acciones: la supervisión, la autoevaluación del/a mediador/a y la retroalimentación del/a supervisor/a, mediante el uso de tres instrumentos, los que dan respuesta a dos modelos teóricos, que son: la mirada de control de desempeño de los mediadores con contrato con el Ministerio de Justicia, y a la mirada de supervisión entre pares, destinada a potenciar el análisis personal de la práctica del/a mediador/a con el apoyo de un experto.

El Índice General de Calidad de la Mediación Familiar permitió concluir que los servicios de los mediadores licitados alcanzan un estándar nacional de 68,28 \%, de calidad alta.

Las sesiones cuya extensión no se prolongó más allá de los 30 minutos se evaluaron mayoritariamente en calidad media, $71,4 \%$. De las sesiones dentro del rango de los 61 a 90 minutos, el mayor porcentaje (50,4\%) se ubicó en calidad 
alta, presentándose diferencias considerables en la distribución de los restantes porcentajes por concepto de calidad, que registraron un 25,2 \% en calidad muy alta, $18,1 \%$ en calidad media y un $6,3 \%$ en baja.

El Coeficiente Rho de Spearman, arroja un valor de 0,276 en la correlación de las variables duración de la sesión y calidad de servicio. Es así como, el estándar de calidad baja tiende a descender a medida que aumenta la duración de las sesiones, desapareciendo sobre 90 minutos.

Análisis nacional de los procesos de mediación:

En cuanto a forma de término, se establecieron tres categorías de término: con acuerdo, en proceso $^{10}$ y frustrada.

Tabla 3. Frecuencia y porcentajes de acuerdo a la forma de término a nivel nacional

\begin{tabular}{ll}
\hline Con acuerdo & $90(32,20 \%)$ \\
\hline Frustrada & $46(16,70 \%)$ \\
\hline En proceso & $143(51,10 \%)$ \\
\hline
\end{tabular}

Fuente: elaboración propia.

Con la finalidad de determinar la existencia de alguna relación entre las variables forma de término y el estándar de evaluación obtenido por concepto del Índice General de Calidad de la Mediación Familiar se calcularon los porcentajes, que muestra la Tabla 4.

Tabla 4. Índice de calidad según duración de la sesión.

\begin{tabular}{lccccc}
\hline & 30 M. o menos & $\mathbf{3 1}$ a $\mathbf{6 0}$ M. & $\mathbf{6 1}$ a 90 M. & $\mathbf{9 1}$ a 120 M. & Más de 120 M. \\
\hline Calidad muy baja & $0,0 \%$ & $0,0 \%$ & $0,0 \%$ & $0,0 \%$ & $0,0 \%$ \\
\hline Calidad baja & $21,43 \%(3)$ & $7,89 \%(6)$ & $6,3 \%$ & $0,0 \%$ & $0,0 \%$ \\
\hline Calidad media & $71,43 \%(10)$ & $34,2 \%(26)$ & $18,1 \%$ & $23,08 \%(12)$ & $12,5 \%(1)$ \\
\hline Calidad alta & $0,0 \%$ & $40,8 \%(31)$ & $50,4 \%$ & $42,31 \%(22)$ & $50,0 \%(4)$ \\
\hline Calidad muy alta & $7,14 \%(1)$ & $17,1 \%(13)$ & $25,2 \%$ & $34,6 \%(18)$ & $37,5 \%(3)$ \\
\hline
\end{tabular}

Fuente: elaboración propia.

\footnotetext{
${ }^{10}$ Para efectos del presente estudio se entiende por sesión o mediación "en proceso", toda aquella en la que no habiéndose logrado un acuerdo entre las partes y no resultando frustrada, ha concluido la sesión bajo la decisión de retomar, el caso en sesiones posteriores.
} 
El Índice General de la Calidad de la Mediación Familiar nacional se ubica en calidad alta, debido a los altos promedios en el índice de legitimidad, que concentró sus medias en $68,28 \%$, en calidad alta y muy alta. No se situó ningún componente en calidad baja, salvo el proceso resolutivo, ubicado dos puntos porcentuales por debajo de tal límite de calidad en el país.

\section{Evaluación ECAME 20I4-20I5}

Para la auditoría de calidad del servicio de mediación familiar en los años 2014-2015, correspondiente al segundo estudio efectuado, la UCEN realizó una investigación aplicada que contempló momentos secuenciales en el tiempo. Entenderemos aquí como investigación aplicada, aquel tipo de estudio que tiene como finalidad, conocer de manera sistemática una situación determinada, en la perspectiva metodológica de lo que Robert Merton denomina estudios de alcance medio (Merton, 2003), es decir, investigaciones con un interés práctico, como es el caso de los diagnósticos donde se investiga para intervenir en la realidad.

\section{Antecedentes metodológicos}

Para esto se organizó un diagnóstico de los resultados de la aplicación de la metodología ECAME de 2011 y 2013, que potencie su mejor aplicación futura, generando una nueva evaluación diagnóstica de tipo descriptiva y evaluativa para la intervención social, que valide un procedimiento e instrumento de supervisión definitivo. Para estos efectos se usó instrumental estadístico cuantitativo, esto es, procedimientos estadísticos relacionados con la descripción de los fenómenos en estudio: distribución de frecuencias, tablas de doble entrada, análisis de media. Además de análisis de correlación de variables, a partir de modelos de regresión lineal (Padua, 1982).

El equipo técnico postuló la necesidad metodológica de confeccionar y aplicar un modelo analítico secuencial de investigación aplicada que para la teoría de investigación exige para su resolución: "[...] además de la reflexión, operaciones tales como la caracterización de fenómenos de observación, enumeración, medición y la construcción de instrumentos, con el uso de miradas paradigmáticas cuantitativas [...]" (Pita y Pértegas, 2002, p. 64), y que en este caso deben concluir con propuestas y sugerencias que permitan potenciar su utilización.

Se usaron herramientas estadísticas para analizar los resultados de la Auditoría de Evaluación de la Calidad de Procesos de Mediación Familiar del 2011, desarrollada por la PUCV y el del año 2013 realizada por la UCEN, permitiendo la interpretación y construcción de insumos para el desarrollo de juicios críticos de expertos/as en mediación que enriquecieran el diagnóstico. Para el diagnóstico se utilizó un enfoque deductivo y analítico de tratamiento de los datos, que se inicia con una 
fase de homologación estadística de las bases de datos para su comparación y para el análisis de los resultados se usó un modelo descriptivo.

El análisis de los resultados del focus group con la presencia de expertos/as en mediación familiar, se realizó mediante la técnica de análisis de contenido, surgiendo como resultado que las capacidades técnico-metodológicas de los mediadores, logran resultados exitosos en circunstancias no favorables, asegurando un proceso de calidad, dado su gran compromiso con los usuarios y el hecho de que en su mayoría están altamente calificados para la función.

Hubo consenso entre los participantes respecto de que la debilidad fundamental de las mediciones se realiza desde la observación hacia el mediador, dejando de lado la observación al usuario. Por otro lado, se reconoció que los usuarios valoran que en el proceso de mediación se discutan contenidos más allá del ámbito de los acuerdos, puesto que éstos llegan a mediación con sus vínculos comunicacionales fragmentados desde larga data (Roché, 2011).

Generalmente, el usuario Ilega a mediación con expectativas bajas, ya que solo conoce la realidad judicial. Necesita de un tercero que despliegue conocimientos teóricos y técnico-metodológicos, que permita ampliar su visión del conflicto (Gavrielides, 2015). Plano en el que hay falencias en la formación del mediador, las que se concentran principalmente en modelos y técnicas.

Se releva como un aspecto importante en el proceso de mediación, cautelar y evaluar permanentemente la salud mental, empatía, aptitudes, atributos humanos del mediador y capacidad de contención, porque eso influye en la calidad de atención del mediador.

Por otra parte, es importante que la mediación se trate con enfoque de género (Lombardo, 2003), aspectos de interculturalidad y derechos de los niños, niñas y adolescentes (Barcia, 2013), para lo que se recomienda aplicar un check list (ONU Mujeres, 2011) que mida este enfoque en el proceso.

\section{Propuesta de pauta ECAME versión 2.0}

De acuerdo con la definición inicial realizada por la CEAL-PUCV, del primer instrumento ECAME, éste corresponde a una metodología de supervisión compleja, que considera el diseño de los componentes en una mirada separada de proceso y de productos, lo que incidió en que se duplicara la evaluación de los ítems, razón por la que se planteó la necesidad de privilegiar la construcción de un enfoque de procesos en el diseño y ordenamiento de los ítems de observación de la pauta de ECAME. 
Así mismo, el instrumento ECAME original contemplaba cuatro escenarios que se aplicaban según la etapa de mediación observada: inicial, intermedia, conjunta, individual o de término con acuerdo, frente a lo que aparece adecuado usar una pauta estandarizada aplicable a cualquier escenario y etapa de la mediación, de modo de equiparar los resultados.

\section{Revisión teórica y juicio de expertos}

Como siguiente etapa, se realizó una mirada teórica de la definición del ECAME creado por CEAL-PUCV, considerando los modelos clásicos en mediación, como la normativa legal vigente; sometiendo la pauta a una primera revisión cualitativa intraequipo, incorporando nuevas categorías de análisis, excluyendo y reordenando algunos fraseos.

Sometiéndose el resultado a un segundo análisis metodológico de juicio de expertos/ as, concluyeron que este instrumento actualizado, compuesto por 44 ítems, favorecía los siguientes criterios: evaluar procesos de trabajo con similar grado de complejidad, independiente de la sesión de que se trate; que pueda ser utilizado por profesionales sin que sea necesario la presencia de especialistas expertos; que el instrumento pueda medir y comparar equitativamente conductas, destrezas y uso de técnicas transversales. Además, la pauta contempló por única vez, una consulta sin puntajes, destinada a evaluar la aplicación de modelos teóricos aplicados en sesión.

Además, se incorporaron a los componentes, ítems complementarios de evaluación, con aspectos relacionales, técnicas de gestión, de contención de emociones y resolución del conflicto y 18 conductas observadas, que pueden ir variando en el tiempo según el énfasis de las políticas públicas ${ }^{11}$, sin focalizarse en modelos de mediación determinados para dar libertad profesional.

\section{Revisión estadística inferencial}

Se realizó un procedimiento de análisis estadístico inferencial para cada ítem, con la finalidad de observar su comportamiento en cuanto a la capacidad de discriminación de la conducta observada y las desviaciones presentes. Para ello, se realizó el cálculo de validez de constructo, entendida como la capacidad que tiene el instrumento de medir estrictamente lo que teóricamente debe medir, en este caso la calidad del proceso de mediación. Ello permitió identificar las variables ocultas, que son subconjuntos jerarquizados del cálculo de la varianza.

\footnotetext{
${ }^{11}$ Para ello y de acuerdo con la opinión de los autores, se sugiere revisar los informes emanados de la UMED, que se caracterizan por el enfoque sistémico que cruza las ciencias sociales y jurídicas y los criterios funcionalistas de la doctrina jurídica chilena en los temas de familia.
} 
En este ejercicio de validez de constructo, entendido como el grado en que el instrumento de medida cumple con las hipótesis que cabría esperar para un instrumento de medida diseñado para medir precisamente aquello que deseaba medir (Castejón, 1997), se obtuvo un nivel KMO 0,883 (nivel bueno), un Chi-cuadrado de 14,64, grados de libertad de 3,25 y una significación de ,000; los que permiten afirmar que existen interrelaciones entre las variables posibles de explicar a través de componentes.

De esta forma, el instrumento arrojó la existencia de 26 ítems distribuidos en tres componentes con distintos tamaños y pesos relativos, todos correlacionados y con alta consistencia entre sí.

El componente 1 es proceso de mediación, el que concentró un total de 22 ítems de los propuestos, que se agrupan en habilidades que despliega el mediador/a, que contienen cuatro subcomponentes: legitimidad, habilidades comunicativas, de participación y gestión del conflicto.

Componente 2: contención de emociones en los mediados, en especial las referidas a agresiones de corte psicológico (Jiménez y Medina, 2011) y labilidad emocional. Componente 3: búsqueda de posibles acuerdos, fortaleciendo la reflexión entre las partes para la toma de decisiones (Veloso, 1999).

Asimismo, existen otros 18 ítems que no correlacionaron estadísticamente entre sí, no correspondiendo al concepto clásico de la mediación en familia. Los que se refieren a aspectos de la política pública, como modificaciones legales (Barcia, 2012); violencia intrafamiliar, co-parentalidad (Lathrop, 2010), presencia de los niños, niñas y adolescentes en la mediación (Villacampa, 2012). Los que por su importancia y haber sido validados por jueces expertos se considera necesario incluirlos, bajo la modalidad de variables observadas.

Lo anterior permitió reconstruir la siguiente definición operativa del ECAME 2,0: La evaluación del abordaje y tratamiento de problemas de índole familiar, donde un tercero/a imparcial, implementa un conjunto de habilidades comunicacionales que potencien la escucha activa entre las personas y herramientas de fomento a la participación activa y responsable de las partes, con la finalidad de apoyar el proceso de identificación de las posturas y necesidades que están presentes en la problemática vivida, enfocando en la búsqueda de posibles soluciones que puedan ser acuerdos de solución respecto del problema que les aqueja, en un ambiente marcado por la contención de desbordes emocionales. 
Un novedoso instrumento para evaluar la calidad de la mediación de conflictos...

Tabla 5. Componentes y factor de saturación.

\begin{tabular}{llll}
\hline \multirow{2}{*}{ Componentes } & Dimensiones & Ítems. \\
\hline \multirow{2}{*}{ Proceso de mediación } & $\bullet$ & Legitimidad & 4 \\
& - & Comunicación & 6 \\
& - & Participación & 5 \\
& & Gestión del conflicto & 3 \\
\hline \multirow{2}{*}{ Contención de emociones } & Detección y control & 4 \\
\hline \multirow{2}{*}{ Búsqueda de posibles acuerdos } & Aclaración y reflexión & 4 \\
\hline \multirow{2}{*}{ Conductas observadas } & - & Legitimidad & 1 \\
& $\bullet$ & Comunicación & 3 \\
& - & Participación & 1 \\
& - & Gestión & 2 \\
& - & Contención de emociones & 1 \\
& $\bullet$ & Resolutivo & 1 \\
\hline
\end{tabular}

Fuente: elaboración propia.

A continuación, se presentan la propuesta de componentes y sub-dimensiones con el factor de correlación que alcanzan entre sí, ordenados de mayor a menor puntaje de correlación.

Tabla 6. Componentes, dimensiones y conductas observadas.

\begin{tabular}{|c|c|c|c|c|c|}
\hline \multicolumn{6}{|l|}{$\begin{array}{l}\text { Componentes y conductas observadas (C.O.) } \\
\text { I: Proceso de mediación }\end{array}$} \\
\hline Dimensión: Legitimidad & MF & $\mathbf{F}$ & $\mathbf{O}$ & MO & A \\
\hline $\begin{array}{l}\text { 1. Da igualdad en el tratamiento de los intereses de las } \\
\text { partes }\end{array}$ & & & & & \\
\hline $\begin{array}{l}\text { 2. Insta a las partes a decidir si desean permanecer en el } \\
\text { proceso de mediación }\end{array}$ & & & & & \\
\hline
\end{tabular}

3. Sus intervenciones refuerzan la neutralidad sin manifestar juicios de valor

4. Favorece la identificación de todos los aspectos de la temática específica a mediar por las partes

\section{$\leftarrow$ 5. Favorece la información jurídica para todas las partes}

6. Facilita el acceso equilibrado de las partes a instancias de asesoría jurídica

7. El lenguaje no verbal es coherente con los principios de neutralidad, imparcialidad y voluntariedad

8. Realiza intervenciones que ponen en relieve las diferencias de género

9. Promueve que los adultos responsables consideren las necesidades de los NNA 


\begin{tabular}{|c|c|c|c|c|c|}
\hline $\begin{array}{l}\text { 11. Promueve el diálogo sobre la participación de ambos } \\
\text { padres en la crianza y desarrollo de los hijos/as (co- } \\
\text { parentalidad) }\end{array}$ & & & & & \\
\hline \multicolumn{6}{|l|}{$\begin{array}{l}\text { 12. Aplica criterios establecidos en leyes y reglamentos } \\
\text { que prohíben mediar en caso de que exista VIF }\end{array}$} \\
\hline \multicolumn{6}{|l|}{$\begin{array}{l}\text { 13. Facilita el conocimiento de las normas sobre alimentos } \\
\text { provisorios. }\end{array}$} \\
\hline \multicolumn{6}{|l|}{$\begin{array}{l}\text { 14. Trabaja conforme a la agenda construida por las } \\
\text { partes }\end{array}$} \\
\hline Dimensión: comunicación & MF & $\mathbf{F}$ & $\mathbf{O}$ & MO & A \\
\hline $\begin{array}{l}\text { 1. Presenta un ritmo y tono de voz apropiado durante } \\
\text { todo el proceso }\end{array}$ & 1 & & & & \\
\hline $\begin{array}{l}\text { 2. Pide aclaraciones ante los mensajes confusos } \\
\text { o contradictorios emitidos por las partes (preguntas } \\
\text { aclaratorias) }\end{array}$ & 1 & & & & \\
\hline $\begin{array}{l}\text { 3. Acompaña con lenguaje analógico sus intervenciones } \\
\text { de manera coherente }\end{array}$ & 2 & & & & \\
\hline $\begin{array}{l}\text { 4. Expresa los mensajes en primera persona para apoyar } \\
\text { la aclaración de relatos de las partes. }\end{array}$ & 2 & & & & \\
\hline $\begin{array}{l}\text { 5. Cuida que las partes respeten las necesidades propias } \\
\text { y la de los otros }\end{array}$ & 3 & & & & \\
\hline $\begin{array}{l}\text { 6. Mantiene permanentemente la distancia física con y } \\
\text { entre las partes }\end{array}$ & 4 & & & & \\
\hline $\begin{array}{l}\text { 7. Utiliza el parafraseo para acoger las afirmaciones de } \\
\text { las partes (parafraseo) }\end{array}$ & 5 & & & & \\
\hline $\begin{array}{l}\text { 8. Resalta los aspectos positivos de los mensajes emitidos } \\
\text { por las partes (connotación positiva) }\end{array}$ & 3 & & & & \\
\hline
\end{tabular}

9. Propicia la circularidad para la mutua comprensión de los mensajes entre las partes (preguntas aclaratorias o preguntas circulares)

\begin{tabular}{llllll}
\hline Dimensión: Participación & MF & F & O & MO & A \\
\hline
\end{tabular}

1. Escucha en forma equitativa a ambas partes, 7 cautelando que sus intervenciones sean equilibradas

2. Permite a las partes decidir sobre si desean o no llegar a acuerdos

\section{7}

3. Intenta mantener el equilibrio del tiempo de expresión de las partes durante el proceso

4

4. Respeta el ritmo de trabajo de las partes 6

5. Abre espacios de participación para las partes de 7 acuerdo al momento del proceso

$\circ$ 6. Propicia la reflexión de las partes respecto de los

$\dot{u} \leftarrow$ posibles efectos de sus posturas en otros (preguntas circulares) 
Un novedoso instrumento para evaluar la calidad de la mediación de conflictos...

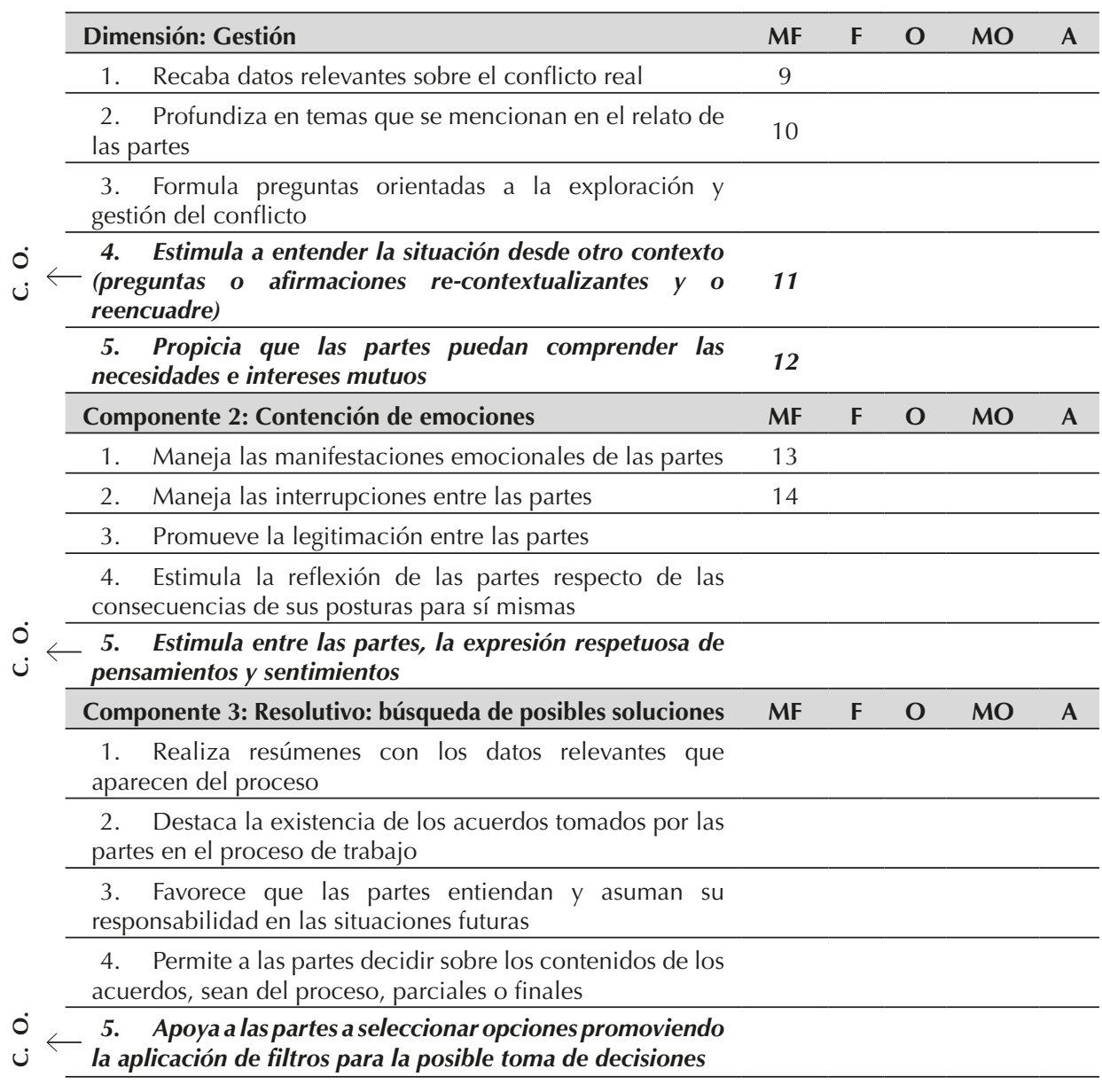

Fuente: elaboración propia.

Finalmente es necesario indicar que el instrumento ECAME 2.0, dispone de un valor de confiabilidad de 0, 897, dado por el "coeficiente de confiabilidad Alfa de Cronbach" (Cronbach, Schoneman, \& Mckie, 1965, p. 65), que se entiende adecuado para la realidad evaluada. Asimismo, se muestran los coeficientes alcanzados por cada componente que dan cuenta de la consistencia interna que muestran.

Estos resultados, al ser confrontados con la definición ampliada del ECAME, permite resaltar la concepción holística que dispone la metodología de calidad, mediante la observación de conductas profesionales complejas en la articulación de la teoría y práctica. 


\section{Cálculo de la pauta ECAME 2.0.}

La pauta ECAME 2.0 está compuesta por dos modalidades de evaluación que son la pauta de ítems correlacionados y la lista de cotejo de conductas con un total de 44 indicadores que, al ser ponderados ordinalmente en escala Likert, entregan la calificación del juicio experto. Dicho cálculo se realiza con la fórmula:

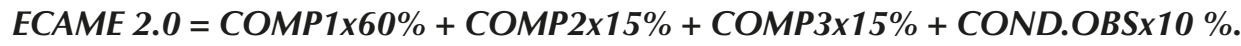

Dichos resultados se traspasan luego a la siguiente categoría de logros, expresada en porcentajes.

Tabla 7. Índice de calidad según categoría.

\begin{tabular}{cc}
\hline Categoría & Índice Calidad M. Familiar \\
\hline Calidad muy baja & $0-25$ \\
\hline Calidad baja & $26-50$ \\
\hline Calidad media & $51-70$ \\
\hline Calidad alta & $71-90$ \\
\hline Calidad muy alta & $91-100$ \\
\hline
\end{tabular}

Fuente: elaboración propia.

\section{Supervisiones con uso de la pauta ECAME 2.0.}

Para implementar la lógica compleja de esta auditoría, se trabajó en terreno para evaluar el desempeño técnico de los mediadores licitados, realizando 100 supervisiones ECAME a un grupo de mediadores seleccionados/as a través de un muestreo aleatorio estratificado ${ }^{12}$.

Esta medición permitió evaluar la pertinencia de los ajustes propuestos al instrumento Cálculo de Índice general ECAME y por componente. El valor de índice General ECAME 2.0 es de 83,91 de logro, ubicándolo en condición de calidad alta en su aplicación 2015.

En cuanto a sus tres componentes: el $\mathrm{N}^{\circ} 1$ se ubica en 90,3 de calidad alta; el componente $\mathrm{N}^{\circ} 2$ en 83,4 en calidad alta; el componente $\mathrm{N}^{\circ} 3$ en 63,6 en calidad media y las conductas observadas en un valor de 77,5, en calidad alta.

\footnotetext{
${ }^{12}$ El uso de la muestra aleatoria estratificada, según región, permitiría que el peso de la evaluación de cada mediador/a supervisado/a corresponda al valor real que tiene en el universo de mediadores de las respectivas regiones supervisadas.
} 
Gráfico 1. Índice general ECAME 2.0 y por componente.

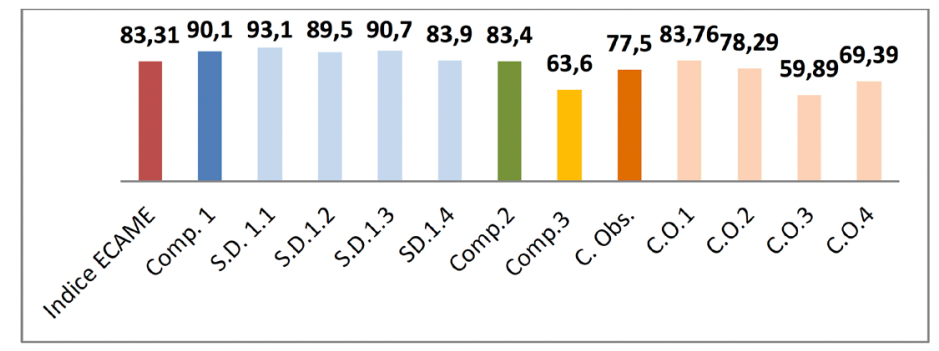

Fuente: base de datos 2015.

Desde una perspectiva comparada en el tiempo, es posible mostrar los resultados de índice general ECAME en los tres momentos de su aplicación, visualizando el aumento progresivo en el alza de los valores que alcanzan los mediadores familiares en materias de mediación previa.

Gráfico 2. Índice general ECAME 2011, 2013 y 2015.

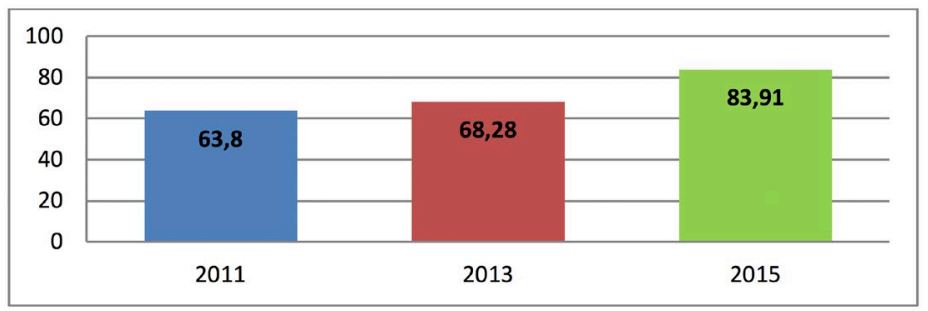

Fuente: elaboración propia.

Sin perjuicio que la diversidad en la configuración del instrumento ECAME con la versión ECAME 2.0 no permite compararlos, se aprecia que este último instrumento permite confirmar que una mejor calidad del proceso depende de variables tales como el desempeño del mediador durante éste, pudiendo observarse, toda vez que el instrumento tendiera a la medición de comportamientos por parte del mediador, que instaran a mantener componentes actitudinales coherentes con los principios y aspectos técnicos de la mediación, por sobre productos específicos, como apareciera en los primeros instrumentos diseñados.

Es así, como la versión 2.0 del instrumento, mide habilidades tales como empatía, aptitudes, atributos humanos del mediador y capacidad de contención, desde un enfoque comportamental visualizado durante el proceso. 
Finalmente, aspectos como incorporación de enfoque de género, aspectos de interculturalidad y derechos de los niños, niñas y adolescentes (señalados como sugerencias en el estudio anterior), se pueden observar y medir con la versión 2.0 del instrumento ECAME durante el proceso observado por los supervisores.

\section{CONCLUSIONES Y SUGERENCIAS}

Es preciso señalar que para el mejoramiento continuo de la calidad del servicio de mediación familiar licitado, el Estado de Chile debe contar con un proceso de evaluación y supervisión de la calidad, estandarizado, revisado y aplicado anualmente a nivel nacional.

En lo referido al Índice General de Calidad de la Mediación, tanto en la primera versión como en el ECAME 2.0, el valor alcanzado lo ubican en el tramo de calidad alta y sobre el corte porcentual de aprobación del mismo identificado en 61 puntos de logro ${ }^{13}$, lo que constituyen un aporte significativo a la valoración del estado de desarrollo de la mediación familiar en Chile luego de diez años de su instalación voluntaria y siete años en calidad de obligatoria.

Algunos aspectos relevantes destacados en los resultados alcanzados son: el análisis bivariado del estudio, con el cruce de las variables de duración de la sesión de la mediación y calidad del servicio, que son de especial importancia, mostrando sus resultados una relación positiva entre la mayor duración de la sesión y una mejor evaluación de calidad, corroborado por el coeficiente Rho de Spearman.

Asimismo, la relación entre la variable género del/a mediador/a y el valor del Índice General de Calidad, informa de una correlación positiva entre el género femenino y los puntajes más altos, que se corrobora con el coeficiente de Chi Cuadrado de Pearson; mediatizado por la alta concentración de mujeres mediadoras frente al bajo número de hombres.

Los tres componentes y sus subcomponentes, que presenta la pauta utilizada en las auditorías, corresponden a estandarizaciones de criterios mínimos de aspectos de contenidos y relacionales, que se deben presentar y desarrollar en un proceso de mediación, con precisiones propias del ámbito familiar, legal y reglamentario, presentes en las conductas observadas.

\footnotetext{
${ }^{13}$ El estudio de la CEAL-PUCV indicó que el puntaje de corte de calidad está puesto en el valor de calidad alta con 61 puntos hacia arriba, para considerar el desempeño del/a mediador/a como adecuado al estándar requerido para la prestación del servicio de mediación, lo que se estima adecuado considerando que coloca el estándar de dificultad en el $60 \%$ de la pauta de evaluación, lo que es similar a las escalas de calificación de conocimientos y de competencias vigentes en Chile del sistema de educarchile.cl.
} 
Desde una lógica evaluativa, la concentración en valores altos alcanzados en este estudio se debe a que se aplicó a los/as mediadores/as que mantienen contrato con el Estado, que son seleccionados mediante una licitación pública, implicando un proceso de selección complejo entre los mejores. Este grupo mantiene una práctica habitual de numerosas mediaciones, siendo permanentemente capacitado, evaluado y supervisado por la UMED, y las que, mediante el proceso de retroalimentación, aportan aprendizaje en la gestión del mediador, permitiéndole un mejoramiento continuo de su praxis. Por esta razón, no es de extrañar que dispongan de una considerable cantidad de herramientas, técnicas y conocimientos conceptuales y procedimentales para un buen estándar de desempeño.

En cuanto al instrumento de evaluación, se puede señalar que, al depurar la versión inicial, en el ECAME 2.0, se ha privilegiado la construcción y diagramación de un enfoque de procesos, distinguiendo las conductas observadas que son variables de los componentes que son permanentes, introduciendo en éstos un conjunto mínimo de técnicas propias de la mediación.

Finalmente, parece importante sugerir que la aplicación de la supervisión debiera contar de periodicidad anual, permitiendo la evaluación constante de los/as mediadores/as que mantienen contrato con el Estado, con igual regularidad que otros funcionarios públicos.

\section{REFERENCIAS BIBLIOGRÁFICAS}

Acuña, M. (2011). Efectos jurídicos del divorcio. Santiago de Chile: Abeledo Perrot y Thompson Reuters.

Aguilar, M. (1994). Introducción a la supervisión. Colección Política, servicio y trabajo social. Buenos Aires, Argentina: Editorial Lumen.

Barcia, R. (2012). Una mirada al cuidado personal y en especial a los artículos 224, 225. $1^{\circ}$ y $3^{\circ}$ del Código Civil y 42 de la Ley de Protección a Menores, desde la perspectiva del Interés Superior de los niños. En Estudios de Derecho Civil VII (X Jornadas de Derecho Civil). Santiago de Chile: Abeledo Perrot, Legal Publishing y Thompson Reuters.

Barcia, R. (2013). Facultades y derechos compartidos respecto de los hijos: una mirada desde el Derecho Comparado. Revista de Derecho Universidad Católica del Norte 20, (1), 21-60.

Beloff, M. (2010). Comportamiento humano y justicia: los equipos multidisciplinarios en las normas internacionales de protección de los derechos de la infancia y su impacto en las legislaciones nacionales latinoamericanas. Fundamentos de psicología jurídica y forense, p. 261- 277. Comp. por Eric García López. Oxford University Press. México, México.

Brandoni, F. (2011). Hacia una mediación de calidad. Buenos Aires, Argentina: editorial Paidós.

Castejón, J. (1997). Introducción a los métodos y técnicas de investigación y obtención de datos en psicología. Sant Vicent del Raspeig, España: ECU.

Correa, P. (2014). La experiencia de la mediación familiar en Chile. Elementos para una política pública futura. Revista Chilena de Derecho y Ciencia Política, 5 (2). 111-138. DOI 10.7770/RCHDYCPV5N2-ART734. 
Cronbach, L., Schoneman, P. \& Mckie, D. (1965). Alpha coefficient for stratified-parallel test. Educ Psychol Meas. Recuperado de http://journals.sagepub.com/doi/10.1177/001316446502500201

Educarchile. (Sin fecha). Escala de calificación conceptual. Recuperado de http://ww2.educarchile.cl/ UserFiles/P0001/Image/pasionxliderar/2011/pdf/competencia5/C5_AC2_R2_ejemplo.pdf

Elna de Jog, E. (2009). Familia. Representaciones y significados. Buenos Aires, Argentina: Espacio.

Fernández, C. y Alarcón, A. (2013). Calidad de la mediación. Teoría versus práctica. Momento, $28(5), 1-18$.

Folger, J. (1993). La promesa de la mediación. Barcelona, España: Gránica.

Gaitán, L. (2006). Sociología de la infancia. Barcelona, España: Ediciones Síntesis.

García, L. (2009). Hijos alineados, padres alienados. Madrid, España: Ediciones Reus.

García, M. y Aguilera, G. (2011). Tres problemas jurídicos asociados al control y supervisión de los mediadores familiares. Revista CREA Universidad Católica de Temuco, pp. 101-115.

Gavrielides, T. (2015). Restorative Justice. The Library of Essays on Justice. Farham: Ashgate Publishing. UK.

Hernández, R. et al. (2003). Metodología de la investigación. México D.F, México: Editorial McGraw-Hill.

Jiménez, M y Medina, P. (2011). Violencia contra la pareja en la justicia penal, mayores penas, mayor violencia. Santiago de Chile: Editorial Librotecnia.

Kerlinger, F. (2002). Investigación del comportamiento. México D.F., México: McGraw-Hill/ Interamericana Editores.

Lathrop, F. (2010). Custodia compartida, acuerdo de los padres y establecimiento de oficio: un fallo en ausencia de la ley. Revista de Derecho, XXIII (2): 414- 474.

Lathrop, F. (2013). Nueva institucionalidad de infancia y adolescencia en Chile. Aportes de la sociedad civil y del mundo académico. Santiago de Chile: Norma.

Lehtinen, U. \& Lehtinen, J. (1982). Service Quality: A Study of Quality Dimensions. The Services Industries Journal, 2 (3), 79-93.

Ley No 19.968 Sobre Tribunales de Familia.

Ley $N^{\circ} 20.260$ Sobre Tribunales de Familia y sus actualizaciones.

Ley $N^{\circ} 20.680$ Sobre Corresponsabilidad Parental.

Lloveras, N. et al. (2010). Los derechos de los niños, niñas y adolescentes. Ediciones Alveroni. Buenos Aires, Argentina.

Lombardo, E. (2003). El Mainstreaming de género en la Unión Europea. Revista Aequalitas, Revista Jurídica de igualdad de Oportunidades entre Mujeres y Hombres, 10-15, 6-11.

López, E. (2016). Mediación y calidad. Recuperado de http://www.cambrabcn.org/documents/ 1533402/1541141/GTM5_TRIBUNA_Mediacion+y+calidad_Emma+Lopez_Ok.pdf?version=1.0.

Merino, C. (2012). La mediación familiar en situaciones asimétricas. Madrid, España: Ediciones Universidad Complutense de Madrid.

Merton, R. (2003). Teoría y estructuras sociales. Madrid, España: Fondo de Cultura Económica de España.

Migrainfo, Diputación de Barcelona. (2008). Balance del espacio de seguimiento y supervisión de la práctica profesional de mediadores y mediadoras comunitarios. Tercer Trimestre. Recuperado de http://www.diba.cat/butlletins/detallRevista.asp?id=6165

Ministerio de Justicia de Chile -AGCI- Unión Europea. (2010). Mediación y resolución colaborativa de conflictos, un aporte a la cohesión social Ministerio de Justicia de Chile. Santiago de Chile.

Montecinos, S. (1991). Madres y Huachos, Alegorías del Mestizaje Chileno. Santiago de Chile: Ediciones Universidad de Chile.

Muñoz, F. (2015) ¿Hacia la academización de las facultades de derecho en Chile? Un análisis teórico y comparado del conflicto de las profesiones. Revista de Derecho (Valdivia), XXVII, 81-95. 
Nash, C. (Comp.). (2014). Derechos Humanos y Mujeres. Teoría y Práctica. Santiago de Chile: Centro de Derechos Humanos. Ediciones Universidad de Chile.

ONU Mujeres. (2011). Checklist Mesa de Género del sistema Naciones Unidas sobre asuntos de género en proyectos. Recuperado de https://www.humanitarianresponse.info/fr/operations/colombia/ document/checklist-mesa-de-g\%C3\%A9nero-del-sistema-naciones-unidas-sobre-asuntos-de.

Ortemberg, O. (2006). Mediación en la violencia familiar y en la crisis de la adolescencia. Buenos Aires, Argentina: Paidós.

Padua, J. (1982). Técnicas de investigación. México: Fondo de Cultura Económica.

Pita, S. y Pértegas, S. (2002). Cuadernos de atención primaria. Recuperado de http://www.fisterra.com/ mbe/investiga/cuanti_cuali/cuanti_cuali.asp.

Preyer, G. (2016) Una interpretación de la globalización: un giro en la teoría sociológica. Revista Mexicana de Ciencias Políticas y Sociales, LXI (226), 61-88.

Rawls, J. (2002). La justicia como equidad: una reformulación. Ed. E. Kelly, trad. Andrés de Francisco. Barcelona, España: Editorial Paidós.

Roché, J. (2011). La mediación y sus principios. Recuperado de www.imet.gob.mx/arts_dirgral/art004.htm.

Rodríguez, M. (2009). El cuidado personal de niños y adolescentes en la familia separada: criterios de resolución de conflictos de intereses entre padres e hijos en el nuevo derecho chileno de familia. Revista Chilena de Derecho, 36 (3): 545-586.

Sallard, S. (2009). Mediación, Supervisión y Contención. Una visión tridimensional. México: Universidad de Sonora.

Serrano, G., López, C., Rodríguez, D. y Mirón, L. (2006). Características de los mediadores y éxito de la mediación. Anuario de Psicología Jurídica, 16, 75-88.

Stein, A. (2015). Contra la prueba libre. Revista de Derecho (Valdivia), XXVII, 245- 261.

Suares, M. (2002). Mediando en sistemas familiares. Buenos Aires, Argentina: Paidós.

Unión Europea (s.f.). Glosario "Mainstreaming y políticas de igualdad de género en Europa". Recuperado de http://www.ucm.es/info/mageeq/glosario.htm.

Universidad de Concepción. (2006). Estudio Calidad del Servicio de Mediación Licitado por el Ministerio de Justicia. Ministerio de Justicia, Santiago de Chile.

Veloso, P. (1999). Igualdad y relaciones familiares. Revista Jurídica de la Universidad de Palermo, Editorial SELA, Buenos Aires, Argentina.

Villacampa, C. (2012). La Justicia Restaurativa en los supuestos de violencia doméstica y de género", en La Justicia Restaurativa, desarrollo y aplicaciones. Granada, España: Editorial Comares. 